\section{Toxoplasmose na gestação: diagnóstico, tratamento e importância de protocolo clínico}

\section{Toxoplasmosis in pregnancy: diagnosis, treatment and the importance of clinical protocol}

Fabiana Burdini Margonato ${ }^{1}$

Ana Mario Rigo Silva ${ }^{2}$

Darli Antonio Soares ${ }^{3}$

Denise Araújo Amaral 4

Airton José Petris ${ }^{5}$

1-3,5 Departamento de Saúde Coletiva. Universidade Estadual de Londrina. Av. Robert Koch, 60. Londrina, PR, Brasil.

CEP: 86.038-440. E-mail: fabianamargonato@yahoo.com.br

4 Central de Abastecimento Farmacêutico de Londrina. Londrina, PR, Brasil

\section{Abstract}

Objectives: to compare therapeutic and laboratory aspects of toxoplasmosis in pregnant women as well as its cost in a real situation and in a hypothetical situation employing a clinical protocol.

Methods: a time-series cohort study was carried out with pregnant women taking part between January and November 2004 in the prenatal program at the Primary Health Units in Londrina, Paraná, Brazil, whose prenatal routine laboratory tests included serology for acute toxoplasmosis. Data were obtained at the city's Department of Health and the University Hospital. Additional tests, treatment and corresponding expenses were compared with data from a hypothetical situation employing a clinical protocol.

Results: in the real situation, there was no definitive diagnosis for $75 \%$ of the pregnant women with reactive IgM for toxoplasmosis, and among those who underwent an additional test, the median time period between the two tests was 23 weeks; $28.5 \%$ of the women were treated without confirmation and a single confirmed case was not treated. Compared with the hypothetical situation, if an additional test had been carried out with the same blood sample it would have been possible to arrive at a definitive diagnosis of all cases. Lower expenditure on medicines would compensate for extra expenses related to additional tests.

Conclusions: the results show the importance of an appropriate clinical protocol both for health protection and cost reduction.

Key words Toxoplasmosis, Treatment protocols, Prenatal diagnosis

\section{Resumo}

Objetivos: comparar aspectos laboratoriais, terapêuticos e gastos com toxoplasmose em gestantes, em época prévia à implantação de protocolo clínico, a uma situação hipotética de aplicação de um protocolo.

Métodos: coorte histórica com gestantes inscritas no pré-natal de unidades básicas de saúde de Londrina, Paraná, que realizaram exames laboratoriais de rotina de pré-natal, incluindo sorologia para toxoplasmose aguda, no período de janeiro a novembro de 2004. Os dados foram obtidos na Secretaria Municipal de Saúde e no Hospital Universitário. A realização de exame confirmatório, tratamento e respectivos gastos foram comparados com dados provenientes de uma situação hipotética de implantação de um protocolo clínico.

Resultados: na situação real, em $75 \%$ das gestantes com IgM reagente para toxoplasmose não houve diagnóstico definitivo e, entre as que realizaram exame confirmatório, o tempo médio decorrido entre os dois exames foi de 23 semanas; $28,5 \%$ foram tratadas sem confirmação e no único caso confirmado não houve tratamento. Na comparação com a situação hipotética, a realização do exame confirmatório na mesma amostra de sangue possibilitaria diagnóstico definitivo a todas. Os menores gastos com medicamentos compensariam o aumento de custos com exames confirmatórios.

Conclusões: os resultados demonstram a importância da implantação de protocolos clínicos de forma adequada, quer seja sob o ponto de vista de proteção à saúde, ou de economia.

Palavras-chave Toxoplasmose, Protocolos de tratamento, Diagnóstico pré-natal 


\section{Introdução}

Segundo estudos ${ }^{1-3}$ realizados no Brasil, a soroprevalência de toxoplasmose na população em geral varia aproximadamente entre 40 e $80 \%$. Apesar desses valores elevados, as maiores preocupações são voltadas às gestantes, devido à possibilidade de infecção congênita, que pode ser muitas vezes grave e até letal. ${ }^{3-7}$

Diante da gravidade da doença congênita, tornase fundamental o início do pré-natal no primeiro trimestre da gestação, possibilitando a identificação precoce dos casos agudos de toxoplasmose gestacional. Ao se diagnosticar precocemente, a realização do tratamento tem maiores chances de evitar ou reduzir seqüelas para o recém-nascido. ${ }^{6,8,9} \mathrm{O}$ Ministério da Saúde preconiza que o tratamento seja feito através da administração de espiramicina, alternada ou não com sulfadiazina, pirimetamina e ácido folínico, dependendo do período gestacional e infecção fetal. ${ }^{10}$

Para o diagnóstico das infecções agudas, a triagem sorológica é a mais indicada, tendo em vista que, na fase inicial, o parasita ainda não pode ser identificado nos tecidos e secreções, o que torna outros métodos, como o isolamento e identificação histológica do Toxoplasma gondii, pouco factíveis para esse momento. ${ }^{7} \mathrm{O}$ marcador sorológico mais freqüentemente utilizado é o anticorpo antitoxoplasma da classe IgM. No entanto, vários autores alertam para a necessidade da realização de exames confirmatórios, como o de avidez de $\mathrm{IgG}$, devido à freqüência elevada de resultado falso-positivo de IgM. ${ }^{7,9,11,12}$

Em Londrina, Estado do Paraná, a triagem sorológica em gestantes é realizada pela Central de Diagnósticos Laboratoriais (CENTROLAB), da Secretaria de Saúde do município, desde 1999, de forma não sistematizada. Na época do estudo não havia protocolo específico para controle desse agravo nas Unidades Básicas de Saúde (UBS). O fluxo observado inicia-se na UBS com a coleta de materiais para exames laboratoriais de rotina de prénatal, que inclui sorologia para toxoplasmose. As amostras são processadas na CENTROLAB e quando há soropositividade de IgM, essa avisa a UBS responsável pela gestante, que coleta nova amostra de sangue, para a repetição do exame de IgM e realização do exame de avidez no laboratório do Hospital Universitário, além providenciar o medicamento junto à Central de Abastecimento Farmacêutico (CENTROFARMA) da Secretaria Municipal de Saúde, que fornece a medicação. Por ser esse um processo complexo a ser percorrido, a falta de um protocolo proporciona que diferentes condutas sejam tomadas entre os próprios profissionais da rede municipal de saúde.

Verificam-se divergências nas condutas relativas ao diagnóstico e tratamento da toxoplasmose aguda gestacional, o que motivou a realização deste estudo. Assim, o objetivo foi comparar aspectos laboratoriais, terapêuticos e gastos com toxoplasmose em gestantes, em época prévia à implantação de protocolo clínico, com os dados consequientes a uma situação hipotética de aplicação de um protocolo, com a finalidade de avaliar as possíveis vantagens da sistematização de condutas através de um protocolo clínico.

\section{Métodos}

Este é um estudo individuado (não agregado), ${ }^{13}$ observacional e longitudinal, retrospectivo (coorte histórica). A população foi constituída pelo total de gestantes inscritas no programa de pré-natal das 53 UBS de Londrina, e que realizaram exames laboratoriais de rotina de pré-natal, incluindo o teste para diagnóstico de toxoplasmose aguda no período de janeiro a novembro de 2004.

A lista das gestantes foi obtida no setor de imunologia da CENTROLAB, tendo sido identificadas aquelas com IgM reagente ou indeterminado para toxoplasmose e anotadas as datas de realização desses exames.

Posteriormente, verificou-se quais gestantes realizaram exames confirmatórios (repetição de $\operatorname{IgM}$ e avidez de $\operatorname{IgG}$ ) no laboratório do Hospital Universitário, as datas da realização dos mesmos e os respectivos resultados. Essa verificação foi estendida até o final do mês de junho de 2005, para contemplar as mulheres que se encontravam no início do período gestacional nos meses de setembro a novembro de 2004.

Quanto ao consumo de medicamentos, os dados foram obtidos na CENTROFARMA. Foram verificadas as gestantes tratadas, a data do início do tratamento, a idade gestacional (em semanas) e os medicamentos dispensados com respectivas quantidades. Esses dados foram processados em planilha do Excel para análise.

Quanto à situação hipotética de implantação de protocolo clínico, os seguintes critérios foram utilizados: a) realização do exame de avidez de IgG na mesma amostra de sangue, após o resultado de IgM reagente ou indeterminado; b) exames realizados na nona semana de gestação, sendo considerados os períodos gestacionais com duração média 
de 40 semanas; c) prevalência de 5,9\% de confirmação de infecção recente (baixa avidez de IgG), encontrada em estudo realizado em Ribeirão Preto, São Paulo, em população de gestantes; ${ }^{14} \mathrm{~d}$ ) em casos de confirmação laboratorial, para prevenir a infecção fetal, tratamento com espiramicina na posologia de dois comprimidos de $500 \mathrm{mg} 3 \mathrm{x} /$ dia entre a $9^{\mathrm{a}}$ e a $13^{\mathrm{a}}$ semana gestacional; espiramicina alternada a cada três semanas com o esquema tríplice, composto de sulfadiazina (seis comprimidos de $500 \mathrm{mg} / \mathrm{dia}$ ), ácido folínico (2/3 de comprimido de $15 \mathrm{mg} / \mathrm{dia})$ e pirimetamina (dois comprimidos de $25 \mathrm{mg} / \mathrm{dia}$ ) entre a $14^{\mathrm{a}}$ e a $34^{\mathrm{a}}$ semana gestacional; e espiramicina na posologia de dois comprimidos de $500 \mathrm{mg} \mathrm{3x/dia} \mathrm{da}$ $35^{\mathrm{a}}$ semana até o termo $\left(40^{\mathrm{a}}\right.$ semana gestacional para este estudo); e) gestantes com peso médio de $60 \mathrm{~kg}$ para o cálculo da dosagem de sulfadiazina. ${ }^{15}$

As variáveis estudadas foram: realização do exame confirmatório, intervalo de tempo entre a data de realização do primeiro exame e a data de realização do exame confirmatório, medicamento(s) dispensado(s) e sua(s) quantidade(s), gasto com diagnóstico laboratorial e com tratamento. Para o cálculo de gastos, os valores dos exames e medicamentos foram obtidos na Diretoria de Ações em Saúde da Secretaria Municipal de Saúde de Londrina no segundo semestre de 2005.

Esta pesquisa foi realizada mediante aprovação prévia do Comitê de Ética em Pesquisa da Universidade Estadual de Londrina (parecer 300/04).

\section{Resultados}

Entre as 4858 gestantes que realizaram exames de pré-natal, $120(2,5 \%)$ apresentaram IgM reagente ou indeterminado para toxoplasmose (Figura 1). Entre estas, aproximadamente $75 \%$ não compareceram para realização de exame confirmatório. O tempo médio entre a realização do primeiro e do segundo exame foi de 9,8 semanas, com períodos que variaram entre 2 e 20 semanas.

\section{Figura 1}

Fluxograma das gestantes que realizaram exames para diagnóstico de toxoplasmose aguda. Londrina, Paraná, janeiro a novembro de 2004.

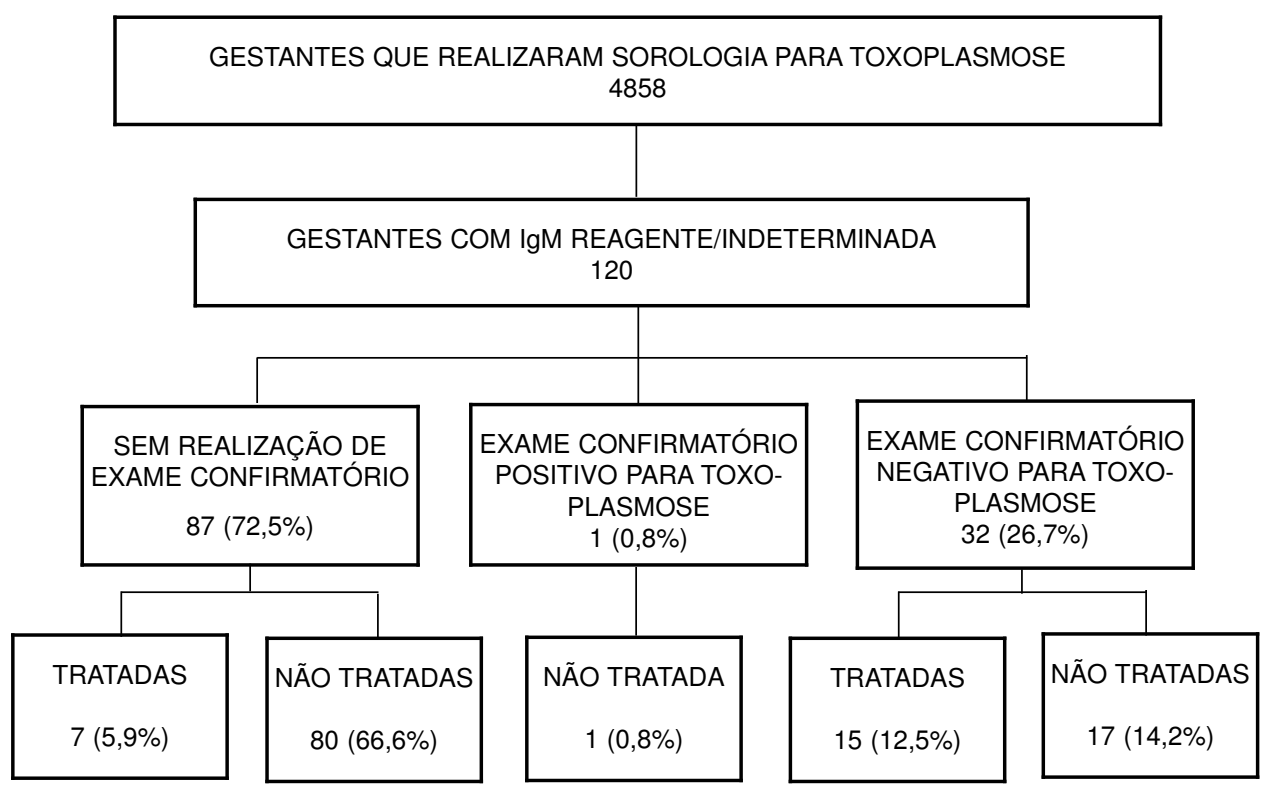


Table 1

Variáveis relacionadas ao diagnóstico e tratamento da toxoplasmose aguda em gestantes, segundo às situações real e hipotética. Londrina, Paraná, janeiro a novembro de 2004.

\begin{tabular}{|c|c|c|c|}
\hline \multirow[t]{2}{*}{ Variável } & \multicolumn{2}{|c|}{ Situação } & \multirow[t]{2}{*}{ Diferença absoluta } \\
\hline & Real & Hipotética & \\
\hline \multicolumn{4}{|l|}{ Exames realizados ( $n^{\circ}$ gestantes): } \\
\hline $\operatorname{lgG} /$ Toxo & 4858 & 4858 & \\
\hline $\lg M / T o x o$ & 4858 & 4858 & \\
\hline Repetição de IgM & 33 & - & -33 \\
\hline Avidez de IgG & 14 & 120 & +106 \\
\hline Gestantes com diagnóstico/ infecção recente & 1 & 7 & +6 \\
\hline Gestantes tratadas & 22 & 7 & -15 \\
\hline Início do tratamento (semanas de gestação - média) & $23^{a}$ & $9^{a}$ & -14 semanas \\
\hline \multicolumn{4}{|l|}{ Comprimidos dispensados $\left(n^{\circ}\right)$} \\
\hline Espiramicina & 11316 & 5964 & -5352 \\
\hline Sulfadiazina & - & 4116 & +4116 \\
\hline Ácido Folínico & - & 462 & +462 \\
\hline Pirimetamina & - & 1372 & +1372 \\
\hline Gasto com medicamentos $(\mathrm{R} \$)^{*}$ & $16.521,36$ & $11.101,16$ & $-5.420,20$ \\
\hline Gasto com exames $(\mathrm{R} \$) * *$ & $181.103,65$ & $182.457,80$ & $+1.354,15$ \\
\hline Gasto total (R\$) & $197.625,01$ & $193.558,96$ & $-4.066,05$ \\
\hline
\end{tabular}

* Custo unitário dos medicamentos ( $R \$)$ : espiramicina $=1,46$; sulfadiazina $=0,08$; ácido folínico $=1,32$; pirimetamina $=0,05$;

** Valores de tabela do Sistema Único de Saúde (SUS) (R\$) para sorologia de IgM, IgG e avidez de IgG=18,55/exame.

Verificou-se que quase $20 \%$ das gestantes foram tratadas sem realização de exame confirmatório ou com resultado que não apontou infecção recente. No entanto, o único caso em que houve confirmação laboratorial de infecção aguda não foi tratado.

A Tabela 1 apresenta a comparação de variáveis levantadas na situação real e as estimadas na situação hipotética criada no presente estudo.

Verificou-se aumento de gastos com exames laboratoriais, porém houve redução dos gastos totais devido a não utilização de medicamentos desnecessários, diferente da situação real. Assim, seriam economizados $\mathrm{R} \$ 4.066,05$ se o diagnóstico e o tratamento fossem realizados de acordo com a situação hipotética.

\section{Discussão}

Destaca-se, nesse estudo, a baixa freqüência de gestantes que realizaram exame confirmatório (avidez de IgG), fato que pode ter sido decorrente de falhas na comunicação entre o laboratório, a unidade básica de saúde e a gestante. Outro fator limitante pode estar relacionado à necessidade de retorno da usuária ao serviço. Esse problema poderia ser total- mente resolvido com a implantação de um protocolo que indicasse a verificação de retorno da gestante imediatamente após detecção de níveis de $\operatorname{IgM}$ reagente ou indeterminado. Por essa razão, em alguns estudos de prevalência de toxoplasmose gestacional, o exame de avidez é realizado com a mesma amostra de sangue. ${ }^{11}$ Essa conduta também é seguida no protocolo clínico da Secretaria Municipal de Saúde de Curitiba ${ }^{15}$ e poderia ser adotada na cidade do estudo, sem aumento de custos.

Além de garantir o cumprimento do diagnóstico integral, a realização do exame de avidez na mesma amostra de sangue, em todos os casos em que o resultado de $\operatorname{IgM}$ for reagente ou indeterminado, é pertinente por reduzir a frequiência de falsos-positivos e evitar danos desnecessários à gestante, como preocupação com a transmissão da doença ao feto e uso desnecessário de medicamentos. ${ }^{11,16}$

A freqüência de baixa avidez de anticorpos IgG para toxoplasmose $(0,8 \%)$ encontrada em Londrina é inferior àquela observada em Ribeirão Preto $(5,9 \%) .{ }^{14}$ Uma das possíveis explicações para esse fato é a realização dos exames de $\operatorname{IgM}$ e avidez de IgG em tempos diferentes, uma vez que o intervalo médio observado até a realização do exame confir- 
matório foi de 9,8 semanas, mas em alguns casos chegou a até 20 semanas. Montoya et al. ${ }^{11}$ apontam que a realização do exame de avidez de $\mathrm{IgG}$ detecta apenas infecções mais recentes do que 12 semanas, o que leva a supor que algumas das gestantes estudadas possam ter tido infecção aguda, sem que o diagnóstico fosse realizado.

A utilização da estimativa de frequiência de 5,9\% de baixa avidez encontrada em Ribeirão Preto ${ }^{14}$ é justificada pela inexistência de trabalhos anteriores realizados em Londrina que estimassem a prevalência de baixa avidez de IgG em gestantes com IgM reagente ou indeterminado para toxoplasmose. Além disso, destaca-se que esse município apresenta características climáticas, econômicas e culturais semelhantes às de Londrina.

O emprego da nona semana gestacional para realização dos exames na situação hipotética deve-se à importância de iniciar o pré-natal no primeiro trimestre de gestação, o que garantiria, entre outras vantagens, o diagnóstico precoce da infecção nesse período. ${ }^{11}$ Entretanto, em estudos brasileiros têm-se observado que parte das gestantes não inicia o prénatal no primeiro trimestre gestacional. ${ }^{17-19}$ Assim, a estimativa de gastos na situação hipotética foi superestimada.

A necessidade do diagnóstico precoce e definitivo se dá frente a resultados obtidos por diversos autores que observaram que, no primeiro trimestre gestacional, o risco de transmissão vertical da toxoplasmose não é tão elevado como nos trimestres posteriores, contrário à gravidade da doença congênita, que é maior quanto mais recente a gestação. ${ }^{6,16,20-22}$ Dessa forma, o diagnóstico precoce auxiliaria principalmente na prevenção dos casos mais graves. ${ }^{21}$

Quanto ao tratamento com droga única, a espiramicina, cabe ressaltar que na maioria dos trabalhos e protocolos existentes, associa-se, ao seu uso, o esquema tríplice: pirimetamina, sulfadiazina e ácido folínico. ${ }^{15,21}$ Segundo Gagne, ${ }^{7}$ a espiramicina previne a passagem transplacentária do toxoplasma para o feto, enquanto os outros medicamentos atra- vessam a barreira placentária tratando diretamente o feto via intra-uterina, prevenindo ou diminuindo seqüelas. Acredita-se que a falta de um protocolo fez com que apenas a espiramicina tivesse sido utilizada na situação real.

Outro fato a ser discutido é a utilização desnecessária de medicamentos em gestantes sem confirmação laboratorial de toxoplasmose. Tal fato pode ser constatado ao se observar que o número de comprimidos gastos foi maior na situação real, que identificou apenas uma gestante com infecção, do que na situação hipotética, na qual sete gestantes estariam infectadas. Se as gestantes da situação real tivessem sido tratadas desde o início da gravidez, a quantidade de medicamentos dispensada seria ainda muito maior. É importante lembrar ainda que, na situação real, a única gestante que teve confirmação laboratorial não recebeu tratamento.

A existência de um protocolo na época do estudo poderia ter evitado possíveis eventos adversos a medicamentos desnecessários, preocupações da gestante com o feto e falta de tratamento em caso de infecção, além da diminuição de gastos. $\mathrm{O}$ aumento de recursos necessários com exames de avidez de IgG seria compensado pela quantidade utilizada de medicamentos, que seria reduzida.

A demonstração de vantagens enfatiza ainda mais a importância do seguimento de protocolos, uma vez que, quando há sucesso em sua implantação, funcionam como facilitadores locais de adesão às boas práticas. ${ }^{23}$ No entanto, há que se cuidar da elaboração e implementação de estratégias como responsabilização da equipe de saúde, respaldo técnico das sociedades científicas e hospitais universitários na elaboração de protocolos e treinamento de profissionais, investimento nas capacitações técnicas e gerenciais, monitoramento de queixas e reclamações de usuários, com pronta resposta das partes envolvidas e atuação dos conselhos de saúde, que favoreçam a adesão dos profissionais e usuários ao protocolo a ser seguido. $^{24,25}$

\section{Referências}

1. Rey LC, Ramalho ILC. Seroprevalence of toxoplasmosis in Fortaleza, Ceará, Brazil. Rev Inst Med Trop São Paulo. 1999; 36: 171-4.
2. Garcia JL, Navarro IT, Ogawa L, Oliveira RC, Garcia SMF, Leite J. Soroepidemiologia da toxoplasmose e avaliação ocular pela tela de Amsler, em pacientes da zona rural, atendidos na unidade de saúde do município de Jaguapitã, PR, Brasil. Rev Soc Bras Med Trop. 1999; 32: 671-6. 
3. Coelho RAL, Kobayashi M, Carvalho Junior LB. Prevalence of IgG antibodies specific to Toxoplasma gondii among blood donors in Recife, Northeast Brazil. Rev Inst Med Trop São Paulo. 2003; 45: 229-31.

4. Reiche EMV, Morimoto HK, Farias GN, Hisatsugu KR, Geller L, Gomes ACLF, Inoue HY, Rodrigues G, Matsuo T. Prevalência de tripanossomíase americana, sífilis, toxoplasmose, rubéola, hepatite $\mathrm{B}$, hepatite $\mathrm{C}$ e da infecção pelo vírus da imunodeficiência humana, avaliada por intermédio de testes sorológicos, em gestantes atendidas de 1996 a 1998 no Hospital Universitário Regional do Norte do Paraná. Rev Soc Bras Med Trop. 2000; 33: 519-27.

5. Bessières MH, Berrebi A, Rolland M, Bloom, MC, Roques C, Cassaing S, Corjaut C, Séguéia JP. Neonatal screening for congenital toxoplasmosis in a cohort of 165 woman infected during the pregnancyand influence of in utero treatment on the results of neonatal tests. Eur J Obstet Gynecol Reprod Biol. 2000; 94: 37-45.

6. Mozzato L, Procianoy RS. Incidence of congenital toxoplasmosis in southern Brazil: a prospective study. Rev Inst Med Trop São Paulo. 2003. 45: 147-51.

7. Gagne SS. Toxoplasmosis. Prim Care Update Ob/Gyns. 2001; 8: 122-4.

8. Urueña CAO, Garcia DFF. Guía de práctica clínica para diagnóstico y manejo de la toxoplasmosis gestacional. Rev Colomb Obstet Ginecol. 2003; 54: 164-70.

9. Montoya JG, Liesenfeld O. Toxoplasmosis. Lancet. 2004; 363: 1965-76.

10. Brasil. Ministério da Saúde. Gestação de alto risco: manual técnico. 3. ed. Brasília, DF; 2001.

11. Montoya JG, Liesenfeld O, Kinney S, Press C, Remington JS. VIDAS test for avidity of toxoplasma - specific immunoglobulin $\mathrm{G}$ for confirmatory testing of pregnant women. J Clin Microbiol. 2002; 40: 2504-8.

12. Lappalainen M, Hedman K. Serodiagnosis of toxoplasmosis. The impact of the measurement of IgG avidity. Ann Ist Super Sanit. 2004; 40: 81-8.

13. Almeida Filho N, Rouquayrol MZ. Elementos de metodologia epidemiológica. In: Rouquayrol MZ, Almeida Filho N. Epidemiologia \& saúde. 6. ed. Rio de Janeiro: Medsi; 2003. p. 149-78.

14. Duarte G. Avaliação de um protocolo de diagnóstico e manejo da toxoplasmose aguda durante a gravidez. $\mathrm{L}$ Congresso Brasileiro de Ginecologia e Obstetrícia; 2003 nov. 18-22; Recife, Pernambuco. Disponível em http://www.febrasgo.org.br. [2005 jan 18].

Recebido em 22 de dezembro de 2005

Versão final apresentada em 11 de julho de 2007

Aprovado em 2 de agosto de 2007
15. Curitiba. Secretaria de Saúde. Programa Mãe Curitibana: prevenção e tratamento das infecções maternas e congênitas. Curitiba; 2004.

16. Ferreira AW, Camargo ME. Toxoplasmosis and the laboratory: diagnosis and a constant striving for improvement. Rev Inst Med Trop São Paulo. 2002: 44: 119-20.

17. Maranhão AGK, Joaquim MMC, Siu C. A mortalidade perinatal e neonatal no Brasil. Brasília, DF: Ministério da Saúde, UNICEF; 1998.

18. Halpernn R, Barros FC, Victora CG, Tomasi E. Atenção prénatal em Pelotas, Rio Grande do Sul, Brasil, 1993. Cad Saúde Pública. 1998; 14: 487-92.

19. Puccini RF, Pedroso GC, Silva EMK, Araújo MS, Silva NN. Equidade na atenção pré-natal e ao parto em área da região metropolitana de São Paulo, 1996. Cad Saúde Pública. 2003; 19: 35-45.

20. Vidigal PVT, Santos DVS, Castro FC, Couto JCF, Vitor RWA, Brasileiro Filho G. Prenatal toxoplasmosis diagnosis from amniotic fluid by PCR. Rev Soc Bras Med Trop. 2002; 35: 1-6.

21. Spalding SM, Amendoeira MRM, Ribeiro LC, Silveira C, Garcia AC, Camillo-Coura L. Estudo prospectivo de gestantes e seus bebês com risco de transmissão de toxoplasmose congênita em município do Rio Grande do Sul. Rev Soc Bras Med Trop. 2003; 36: 483-91.

22. Mombro M, Perathoner C, Leone A, Buttafuoco V, Zotti C, Lievre MA, Fabris C. Congenital toxoplasmosis: assesment of risk to newborns in confirmed and uncertain maternal infection. Eur J Pediatr. 2003; 162: 703-6.

23. Polanczyck CA. Aplicando protocolos na doença cardiovascular. Arq Bras Cardiol. 2004; 82: 307-8.

24. Carvalho DS, Novaes HMD. Avaliação de implantação de programa de pré-natal no Município de Curitiba, Paraná, Brasil. Estudo de coorte em primigestas. Cad Saúde Pública. 2004; 20: 5220-30.

25. Jimenez EJB, Simão MBG, Shimazaki ME. Programa Mãe Curitibana: avançando na humanização da atenção materno-infantil. Divulg Saúde Debate. 2003; 26: 21-9. 